\title{
A Superposition Principle in Quantum Logics
}

\author{
Sylvia Pulmannová \\ Institute for Measurement Theory, Slovak Academy of Sciences, 88527 Bratislava, ČSSR
}

\begin{abstract}
A new definition of the superposition principle in quantum logics is given, which enables us to define the sectors. It is shown that the superposition principle holds only in the irreducible quantum logics.
\end{abstract}

There are several formulations of the superposition principle in physics $[1,2,3]$. In the quantum logic approach to quantum mechanics Gudder [4] postulated that the superposition principle in quantum logic $(L, M)$ holds, if there is an isomorphism between the logic $L$ and the set $\mathscr{M}$ of all "closed" subsets of the set of states $M$.

We shall try to find another formulation of the superposition principle, which will enable us to define the "sectors" analogical to those defined in the $C^{*}$-algebras [3].

First we shall give definitions of the basic concepts. We shall use the expression "quantum logic" in the same sense as in [4].

Let $L$ be a logic, that is an orthocomplemented partially ordered set with the first and last elements 0 and 1, respectively, which has the following orthomodularity property:

$a \leqq b, a, b \in L$ implies that there is a $d \in L, d \leqq a^{\prime}$, such that $b=a \vee d$; and in which $\vee a_{i} \in L$ for all sequences $\left\{a_{i}\right\}$ of mutually disjoint elements in $L$.

The orthocomplementation $a \rightarrow a^{\prime}$ in $L$ has the following properties:

(i) $\left(a^{\prime}\right)^{\prime}=a$; (ii) $a \leqq b$ implies $b^{\prime} \leqq a^{\prime}$; (iii) $a \vee a^{\prime}=1$, where $a, b \in L$.

The elements $a, b \in L$ are disjoint, written $a \perp b$, if $a \leqq b^{\prime}$.

The elements $a, b \in L$ are compatible, written $a \leftrightarrow b$, if there are $a_{1}, b_{1}, c$ in $L$, mutually disjoint and such that $a=a_{1} \vee c$ and $b=b_{1} \vee c$. We shall assume that if $a, b, c$ are mutually compatible, then $a \leftrightarrow b \vee c$.

A map $m: L \rightarrow[0,1]$ which satisfies

(1) $m(1)=1$,

(2) $m\left(\vee a_{i}\right)=\sum_{i} m\left(a_{i}\right)$

if $\left\{a_{i}\right\}$ is a sequence of mutually disjoint elements of $L$, is a state on $L$. If $m$ is a state which cannot be written in the form $m=c m_{1}+(1-c) m_{2}$, where $0<c<1$ and $m_{1}, m_{2}$ are distinct states, then $m$ is called a pure state. Let $M$ be a set of states 
and $P$ the set of all pure states in $M$. If $a \in L, m \in P$, we define $P_{a}=\{m \in P: m(a)=1\}$, $L_{m}=\{a \in L: m(a)=1\}$. As in [4], we call $(L, M)$ a quantum logic if $P_{a} \subset P_{b}$ implies $a \leqq b$, and $L_{m_{1}} \subset L_{m_{2}}$ implies $m_{1}=m_{2}$. If $p, q \in M$, then $m_{0} \in M$ is a superposition of the states $p, q$ if $p(a)=0, q(a)=0$ imply $m_{0}(a)=0$.

Definition 1. A set $S \subset P$ will be said to be closed under superpositions if it contains every pure superposition of any pair of its elements.

If $S$ is not closed under superpositions, let $\Lambda(S)$ denote the smallest subset of $P$ closed under superpositions and containing $S$.

Definition 2. We say that a superposition principle holds in $(L, M)$ if there is an $r \in \Lambda(\{p, q\}), r \neq p, r \neq q$ for any pair $p, q$ in $P, p \neq q$.

That is, a superposition of every two distinct pure states always produces a new pure state.

We shall say that the pair $p, q \in P$ allows only the classical superpositions if $\Lambda(\{p, q\})=\{p, q\}$. That is, a superposition of $p, q$ does not produce any new pure state.

From the foregoing we see that our definition of the superposition principle is different from Guder's definition.

For $S \subset P$ Gudder [4] has defined $\bar{S}$ as the set $\bar{S}=\{r \in P: S(a)=0 \Rightarrow r(a)=0\}$, where he writes $S(a)=0$ if $m(a)=0$ for all $m \in S$. It is shown that if $S=\emptyset$ or $S=\{p\}$, then $\bar{S}=S$.

Theorem 1. The map $S \rightarrow \Lambda(S)$ has the following properties:

1. If $S=\{p, q\}$, then $\Lambda(S)=\bar{S}$.

2. If $S_{1} \subset S_{2} \subset P$, then $\Lambda\left(S_{1}\right) \subset \Lambda\left(S_{2}\right)$.

3. If $S_{\alpha} \subset P, \alpha \in A$, then $\bigcap_{\alpha} \Lambda\left(S_{\alpha}\right)$ is closed under superpositions and $\Lambda\left(\bigcap_{\alpha} S_{\alpha}\right) \subset$ $\bigcap_{\alpha} \Lambda\left(S_{\alpha}\right)$

4. If $S_{\alpha} \subset P, \alpha \in A$, then $\bigcup_{\alpha} \Lambda\left(S_{\alpha}\right) \subset \Lambda\left(\bigcup_{\alpha} S_{\alpha}\right)$.

Proof. 1. If $r, s \in \overline{\{p, q\}}$ and $m \in \overline{\{r, s\}}$, then if $p(a)=0, q(a)=0$ there is also $r(a)=0$, $s(a)=0$, which implies $m(a)=0$. That is $m \in \overline{\{p, q\}}$ and $\overline{\{r, s\}} \subset \overline{\{p, q\}}$, from which it follows that $\overline{\{p, q\}}$ is closed under superpositions; therefore $A\{p, q\} \subset \overline{\{p, q\}}$. On the other hand, $\{p, q\} \subset \Lambda(p, q\}$ is evident.

2. If $S_{1} \subset S_{2}$, then $S_{1} \subset \Lambda\left(S_{2}\right)$, and $\Lambda\left(S_{1}\right) \subset \Lambda\left(S_{2}\right)$ follows from the minimality of $\Lambda\left(S_{1}\right)$.

3. $S_{\alpha} \subset \Lambda\left(S_{\alpha}\right)$ for every $\alpha$, this implies $\bigcap_{\alpha} S_{\alpha} \subset \bigcap_{\alpha} \Lambda\left(S_{\alpha}\right)$. As $\bigcap_{\alpha} \Lambda\left(S_{\alpha}\right)$ is clearly closed under superpositions, we have $\Lambda\left(\bigcap_{\alpha} S_{\alpha}\right) \subset \bigcap_{\alpha} \Lambda\left(S_{\alpha}\right)$.

4. $S_{\alpha} \subset \bigcup_{\alpha} S_{\alpha}$ imply $\Lambda\left(S_{\alpha}\right) \subset \Lambda\left(\bigcup_{\alpha} S_{\alpha}\right)$ by the property 3 , and from this we have
$\bigcup_{\alpha} \Lambda\left(S_{\alpha}\right) \subset \Lambda\left(\bigcup_{\alpha} S_{\alpha}\right)$. 
Definition 3. The set $S \subset P$ is a sector if

1. $S=\Lambda(S)$;

2. If $p, q \in S$, then there is an $r \in \Lambda(\{p, q\}), r \neq p, r \neq q$;

3. If $q \in P, q \notin S$, then $\Lambda(\{p, q\})=\{p, q\}$ for any $p \in S$.

Roughly speaking, a sector is the maximal subset of $P$ closed under superpositions, for which the superposition principle holds.

Theorem 2. If $S_{1}, S_{2} \subset P, S_{1} \neq S_{2}$ are sectors, then $S_{1} \cap S_{2}=\emptyset$.

Proof. Let there exist $p, q \in P$ such that $p \in S_{1}, p \in S_{2}$ and $q \in S_{1}, q \notin S_{2}$. Then $p \in S_{1}$, $q \in S_{1}$ imply the existence of an $r \in \Lambda(\{p, q\}), r \neq p, r \neq q$; while $p \in S_{2}, q \notin S_{2}$ imply $\Lambda(\{p, q\})=\{p, q\}$.

It can be seen from [4] and from Theorem 1, 1. that in the separable classical logic there are no sectors.

Let $C$ be the set of all elements of $L$ which are compatible with all the other elements, that is $C=\{a: a \leftrightarrow b$ for all $b \in L\}$. $C$ is called the center of $L$. Varadarajan [5] has shown that $C$ is a Boolean sub- $\sigma$-algebra of $L$.

A logic $L$ is called irreducible if its center consists only of 0 and 1 .

If $p$ is a pure state and $c \in C$, then $p(c)=1$ or $p(c)=0[6]$.

Theorem 3. If the superposition principle in the quantum logic $(L, M)$ holds, then $L$ is irreducible.

Proof. Let $c \in C, c \neq 0, c \neq 1$. Let $p, q \in P$ and $p(c)=1, q(c)=0$. Let $r \in \Lambda(\{p, q\})$. Since $a \leftrightarrow c$ if $a \in L$, we can write $a=(a \wedge c) \vee\left(a \wedge c^{\prime}\right)$. From $p\left(c^{\prime}\right)=0$ we have $p\left(a \wedge c^{\prime}\right)=0$, that is $p(a)=p(a \wedge c)$. Analogically $q(c)=0$ implies $q(a)=q\left(a \wedge c^{\prime}\right)$ for all $a \in L$. As $r \in P$, we have $r(c)=1$ or $r(c)=0$. Let $r(c)=1$; then $r(a)=r(a \wedge c)$ for all $a \in L$. If $b \in L, p(b)=0=p(b \wedge c)$, then also $r(b)=r(b \wedge c)=0$, because $q(b \wedge c)=0$ for all $b \in L$ and $r$ is the superposition of $p$ and $q$. Thus we have $L_{p} \subset L_{r}$, which implies $p=r$. If $r(c)=0$, then $r\left(c^{\prime}\right)=1$ and we can show in the same way that $q=r$. Thus we have found the states $p, q \in P$ such that $\Lambda(\{p, q\})=\{p, q\}$ and the superposition principle does not hold. The converse statement to Theorem 3 does not seem to be so easy to prove. Let us prove it for a special case.

Theorem 4. Let $(L, M)$ be a quantum logic. If

1. L is irreducible;

2. L satisfies Piron's axioms [7];

3. $p(a)=1, p(b)=1$ imply $p(a \wedge b)=1$ for all $p \in P$ and $a, b \in L$, then the superposition principle holds in $(L, M)$. We note that assumption 3 holds both in the Hilbert space logic and the separable classical logic.

Proof. If $L$ is irreducible and satisfies Piron's axioms, then for any pair $u, v$ of atoms in $L$ there is an atom $z \in L$ such that $z \leqq u \vee v$.

If $a \in L, a \neq 0$, then there must be at least one state $m \in P$ with $m(a)=1$. If it is not so, then from $P_{a}=\emptyset$ we have $P_{a}=P_{0}$, from which it follows that $a=0$. Let $p \in P_{u}$ and $q \in P_{v}$. Then $p \neq q$, because if $p=q$, then from assumption 3 it follows that $p(u \wedge v)=1$, which is impossible for $u \wedge v=0$. Let $r \in P_{z}$, then $r \neq p, r \neq q$ for the same reasons. If $a \in L, p(a)=1, q(a)=1$, then also $p(u \wedge a)=1, q(v \wedge a)=1$. 
However, $u \wedge a=0$ or $u$, because $u$ is an atom. Hence $u \wedge a=u$, that is $u \leqq a$. Analogously $v \leqq a$ and $z \leqq u \vee v \leqq a$ imply that $r(a)=1$. Thus we have shown that $r \in \Lambda(\{p, q\})$.

The center $C$ of a logic $L$ is discrete if there exists an at most countable set $\left\{c_{n}\right\}_{n \in D}$ of mutually disjoint elements of $C$ such that (i) $\bigvee_{n} c_{n}=1$, (ii) $C$ consists precisely of all the lattice sums $\bigvee_{n \in Z} c_{n}$, where $Z$ is an arbitrary subset of $D$. The $c_{n}-s$ are called the atoms of $C$.

Let $L$ be a logic with the discrete center $C$. Then given any $a \in L$ we can write $a=a_{1} \vee a_{2} \vee \ldots$, where $a_{j} \leqq c_{j}$ and the $a_{j}$ are uniquely determined (in fact $a_{j}=a \wedge c_{j}$ ). If we define $L_{j}=L_{\left[0, c_{j}\right]}=\left\{b: b \in L, b \leqq c_{j}\right\}$, then $L_{j}$ are irreducible logics $\left(c_{j}\right.$ is the first element of $L_{j}$ ). The logic $L$ may thus be thought as a direct sum of the irreducible logics $L_{j}$. Varadarajan [6] proved the following statement:

Theorem 5 (Varadarajan). Let $L$ be any logic and $C$ its center. Let $P$ be the set of pure states of $L$. Suppose that $C$ is discrete and that $\left\{c_{n} ; n \in D\right\}$ is the family of atoms of $C$. Then for each $n \in D, L_{\left[0, c_{n}\right]}=L_{n}$ is an irreducible logic. If $p$ is a pure state of $L_{n}$ and we define $\tilde{p}$ on $L$ by

$$
\tilde{p}(a)=p\left(a \wedge c_{n}\right) \quad(a \in L),
$$

then $\tilde{p}$ is a pure state of $L$. If

$$
P_{n}=\left\{\tilde{p}: p \text { a pure state of } L_{n}\right\},
$$

then the $P_{n}: n \in D$ are disjoint subsets of $P$ and $P=\bigcup_{n \in D} P_{n}$.

Let the assumptions of Theorem 5 be satisfied. Let $\tilde{p}, \tilde{q} \in P_{n}$ for some $n \in D$ and $r \in \Lambda(\{p, q\})$. Then $\tilde{p}\left(c_{n}\right)=1, \tilde{q}\left(c_{n}\right)=1$ imply $r\left(c_{n}\right)=1$, while $\tilde{p}\left(c_{m}\right)=0, \tilde{q}\left(c_{m}\right)=0$ imply $r\left(c_{m}\right)=0$ for every $m \neq n$. Then for any $a \in L$ we have $r(a)=r\left(\bigvee_{j} a \wedge c_{j}\right)=$ $\sum_{j} r\left(a \wedge c_{j}\right)=r\left(a \wedge c_{n}\right)$. From this we see that $r \in P_{n}$ and therefore $\Lambda\left(P_{n}\right)=P_{n}$ for all $n \in D$.

On the other hand if $\tilde{p} \in P_{n}$ and $\tilde{q} \in P_{m}, m \neq n$, then $\tilde{p}\left(c_{n}\right)=1, \tilde{q}\left(c_{n}\right)=0$ and from the proof of Theorem 3 it follows that $\Lambda\left(\left\{p^{\sim}, q^{\sim}\right\}\right)=\left\{p^{\sim}, \tilde{q}\right\}$. Thus we have proved the following statement.

Theorem 6. Let $(L, M)$ be a quantum logic, where $L$ is a logic with the discrete center and let $P=\cup P_{n}$. Then

1. $\Lambda\left(P_{n}\right)=P_{n}$;

2. $p \in P_{n}, q \in P_{m}, m \neq n$ imply $\Lambda(\{p, q\})=\{p, q\}$.

Let $L$ be a logic with the discrete center $C$; the $L$ can be written as the direct sum of the logics $L_{i}=L\left[0, c_{i}\right]$. Let $M$ be a set of states on $L$. Then, analogously as in [8], we can find to each $m \in M$ the states $m_{i}$ on $L_{i}$ such that

$$
m(b)=\sum_{\left\{i: m\left(c_{i}\right) \neq 0\right\}} m_{i}\left(b \wedge c_{i}\right) m\left(c_{i}\right) \quad(b \in L) .
$$


It is sufficient to set

$$
m_{i}\left(b \wedge c_{i}\right)=m\left(b \wedge c_{i}\right) / m\left(c_{i}\right) \text { if } \quad m\left(c_{i}\right) \neq 0 .
$$

If $m\left(c_{i}\right)=0$, then we can set $m_{i}(\cdot)=0$. Let $M_{i}$ be the sets of all such $m_{i}$. It is evident that if $\tilde{p} \in P_{i}$, then $p$ is a pure state in $M_{i}$.

Theorem 7. If $(L, M)$ is a quantum logic, it follows that $\left(L_{i}, M_{i}\right), i \in D$ are quantum logics as well.

Proof. If $a \in L_{i}, m$ is a pure state on $L_{i}$, then let $P_{a}^{i}=\left\{m\right.$ a pure state on $\left.L_{i}: m(a)=1\right\}$, $L_{m}^{i}=\left\{a \in L_{i}, m(a)=1\right\}$. We have to show that $P_{a}^{i} \subset P_{b}^{i}$ implies $a \leqq b$ and $L_{m_{1}}^{i} \subset L_{m_{2}}^{i}$ implies $m_{1}=m_{2}$. Let $\tilde{m} \in P$, then $\tilde{m} \in \bigcup_{i} P_{i}$. If $\tilde{m} \in P_{j}, j \neq i$, then $\tilde{m}(a)=0$ for $a \in L_{i}$. Therefore $\tilde{m} \in P_{a}$ if and only if $m \in P_{a}^{i}$, that is $P_{a}^{i} \subset P_{b}^{i}$ implies $P_{a} \subset P_{b}, a, b \in L_{i}$.

If $a \in L_{m}^{i}, m$ is a pure state on $L_{i}$, then $\tilde{m}(a)=m\left(a \wedge c_{i}\right)=1$, that is $a \in L_{\tilde{m}}$. On the other hand, if $a \in L, a \in L_{\tilde{m}}$, than $\tilde{m}(a)=m\left(a \wedge c_{i}\right)=1$, that is $a \wedge c_{i} \in L_{m}^{i}$. Thus $L_{m_{1}}^{i} \subset L_{m_{2}}^{i}$ implies $L_{\tilde{m}_{1}} \subset L_{\tilde{m}_{2}}$, that is $m_{1}=m_{2}$.

From Theorems 6 and 7 it follows that if the irreducible quantum logics $\left(L_{i}, M_{i}\right)$ are such that the superposition principle holds in them, then $P_{i}$ are exactly the sectors in $P$.

\section{References}

1. Dirac, P. A. M.: Principy kvantovoj mechaniki. Gosudarstvennoje izdatel'stvo fiziko-matematičeskij literatury. Moskva 1960

2. Jauch, J. M.: Foundations of Quantum Mechanics. Reading, Mass.: Addison-Wesley 1968

3. Roberts, J.E., Roepstorff, G.: Commun.-Math. Phys. 11, 321 (1969)

4. Gudder, S.P.: J. Math. Phys. 11, 1037 (1970)

5. Varadarajan, V.S.: Commun. Pure Appl. Math. XV, 189 (1962)

6. Varadarajan, V.S.: Geometry of quantum theory. Princeton, N.Y.: Van Nostrand 1968

7. Piron, C.: Helv. Phys. Acta 37, 439 (1964)

8. Gudder, S. P.: Pac. J. Math. 19, 81 (1966)

Communicated by R. Haag

Received October 27, 1975 
